\title{
14 Jahre in der Redaktion der Zeitschrift für Kinder- und Jugend- psychiatrie und Psychotherapie - ein Blick in die Zukunft
}

\author{
14 years in the editorial office of the German Journal \\ of Child and Adolescent Psychiatry and Psychotherapy - \\ a glimpse into the future
}

\author{
Beate Herpertz-Dahlmann \\ Klinik für Psychiatrie, Psychosomatik und Psychotherapie des Kindes- und Jugendalters, Aachen
}

Das letzte Heft dieses Jahres widmet sich u.a. der Geschichte unseres Faches: der Beitrag von Helmut Remschmidt (2018) zeichnet die Erfolgsgeschichte unserer eigenständigen Facharztdisziplin auf.

Seit 45 Jahren gibt es die Zeitschrift für Kinder- und Jugendpsychiatrie und -psychotherapie, die wesentlich zur Identitätsfindung unseres Faches und seiner Vertreter beigetragen hat. Ich hatte das Glück, an der Gestaltung dieser Zeitschrift 14 Jahre lang mitwirken zu dürfen, davon fünf Jahre als Schriftleiterin und neun Jahre als Herausgeberin. Schaut man sich die Editorials vor 14 Jahren an, haben sich die Themen erstaunlich wenig geändert, so z. B. das Editorial zu ,Kinder- und Jugendpsychosomatik" von April 2005 (Schulte-Markwort \& Lehmkuhl 2005) oder „Auf dem Weg zu einer Europäischen Kinderund Jugendpsychiatrie“ von Dezember 2005 (Rothenberger 2005).

In der Zeit meiner Tätigkeit für die Zeitschrift waren Form und Inhalt der Zeitschrift prägenden Veränderungen unterworfen, u. a. durch die Zunahme der Artikel, die sich in der Anzahl der Hefte/Jahr manifestierte, und durch die Etablierung eines eigenen politischen Teils, der die enge Verbindung zu unserer Fachgesellschaft herausstellt.

Helmut Remschmidt definiert in seinem Artikel aktuelle und zukünftige Herausforderungen an unsere Disziplin, die sich aus meiner Sicht in folgender Weise darstellen:

\section{Wissenschaftliche Probleme und Zielvorstellungen}

- Viele Fragestellungen zur Prävalenz, Ätiologie und Therapie einer psychischen Störung im Kindes- und Jugendalter werden wir nur lösen können, wenn sich Kliniken zu ihrer Erforschung zusammentun. Diese Zeitschrift hat im letzten Jahr die Arbeiten von Katharina Bühren und Manuel Föcker ausgezeichnet, deren Ergebnisse auf dem bundesweiten Anorexia nervosa-Register, bei dem sich zahlreiche Universitäts- und andere Kliniken zusammengeschlossen haben, beruhen (Bühren et al. 2017). Wir werden wahrscheinlich mehrere solch störungsspezifischer Register brauchen, um zu verlässlichen Aussagen bezüglich kinder- und jugendpsychiatrischer Versorgungslandschaften und Behandlungsmethoden in Deutschland zu kommen. Leider gestaltet sich die Zusammenarbeit und Produktivität in einem Register aufgrund der „Polyphonie“ der vielen Beteiligten, unterschiedlicher Interessen und fehlender finanzieller Ressourcen nicht immer einfach. Hier bedarf es intensiver Diskussion, aber auch der Übung in „Gemeinschaftsprojekten“. Viele von uns sind in erster Linie Einzelkämpfer; die BMBF-Verbände haben hier nur bedingt Abhilfe geschaffen.

- Neben der Zusammenarbeit untereinander bedarf es auch der intensiven Zusammenarbeit mit anderen Diszi- 
plinen. Hierzu gehören nicht nur die Erwachsenenpsychiatrie mit der viel zitierten Perspektive auf die Lebensspanne, die Pädiatrie zur Erforschung psychosomatischer Zusammenhänge, und die Psychologie, sondern vor allem auch die Grundlagenwissenschaften, um neurobiologische Zusammenhänge besser erfassen zu können. Nur so können wir Anschluss an translationale Forschung finden, die für die Aufklärung der Ätiologie vieler psychischer Störungen und die Entwicklung neuer Therapiemethoden erforderlich ist.

- Helmut Remschmidt nennt in seinem Artikel die Notwendigkeit der Therapieforschung. Dies gilt nicht nur für die Psychopharmakatherapie, sondern auch für die Psychotherapie, letztere nicht mehr nur als Behandlungsform, die „Auge in Auge“ in der Sitzung von Psychotherapeut zu Patient stattfindet, sondern möglicherweise auf der Basis ganz neuer Behandlungswege, z. B. mit telemedizinischen oder online-Methoden, um eine größere Anzahl von Patienten versorgen zu können.

- Ein weiteres wichtiges von Helmut Remschmidt genanntes Problem, das von uns in der allernächsten $\mathrm{Zu}$ kunft gelöst werden muss, ist die Nachwuchsförderung. Dabei geht es einerseits um den wissenschaftlichen, andererseits um den klinischen Nachwuchs. Wesentliche Forschung zu Erkrankungen aus unserem Fachgebiet finden mittlerweile durch andere Disziplinen statt, z.B. durch die Erwachsenenpsychiatrie. In der jüngsten Zeit ist u.a. von der DFG der Begriff des "Clinician Scientist“ geprägt worden, der die (befriedigende) Verbindung von klinischer und Forschungstätigkeit bezeichnet. In den letzten Jahren ist eher ein Rückgang der Zahl von Ärzten/Ärztinnen festzustellen, die sich einer solchen Herausforderung stellen. Dies hängt mit der zeitlichen Belastung durch die Anforderungen in der Klinik, aber auch mit den hohen methodischen Anforderungen in der Forschung und einer veränderten Lebenseinstellung (work-life-balance) zusammen. Umso erfreulicher war der Bericht in „European Child \& Adolescent Psychiatry" über die ESCAP Research Academy im letzten Jahr in Genf, wo alle Ärztinnen/Ärzte die Verbindung von Klinik und Forschung anstrebten, während sich die beteiligten Psychologen auch eine ausschließliche Forschungstätigkeit vorstellen konnten (Revet et al. 2018). Aus meiner Sicht wird sich das Problem einer unzureichenden Nachwuchsrekrutierung nicht lösen lassen, wenn nicht zusätzliche Gelder in die Universitätskliniken oder andere forschende Institutionen fließen, um Forschungszeiten zu finanzieren. Während meiner Tätigkeit als Leiterin einer universitären Kinder- und Jugendpsychiatrie ist der ökonomische Druck immer größer geworden, der kaum Freistellungen von Mitarbeitern für Forschungstätigkeit erlaubt. Wir sollten uns gemeinsam dafür einsetzen, dass auch die politischen Gre- mien die Notwendigkeit der Nachwuchsförderung erkennen und neue Modelle zu deren Finanzierung entwickelt werden. Hierfür gibt es bereits Beispiele in unseren Nachbarländern (Schweiz).

\section{Klinische Probleme und Zielvorstellungen}

- Aufnahme der Kinder-und Jugendpsychiatrie in das Curriculum: wie bereits aus den Ausführungen von Helmut Remschmidt hervorgeht, wäre die Integration unseres Faches in die Approbationsordnung ein wichtiger Schritt für die Nachwuchsgewinnung. Solange Studenten nicht bereits während des Studiums mit unserem Fach in Berührung kommen, gestaltet sich die Akquise von Assistenzärzten für die Weiterbildung schwierig. Bisher sind leider alle Bemühungen - auch durch Studiendekane aus unserem Gebiet - gescheitert, die Kinderund Jugendpsychiatrie in das Medizinstudium aufzunehmen. Dies ist nicht nachzuvollziehen, da $10 \%$ aller Kinder und Jugendlichen in Deutschland eine behandlungsbedürftige psychische Störung aufweisen.

- Obwohl die Psychotherapie Teil unserer Gebietsbezeichnung ist, gerät sie in der Facharztweiterbildung mehr in den Hintergrund. Durch den Ärztemangel an vielen Kliniken werden die Ärzte zunehmend für rein medizinische Aufgaben „abgestellt“, während die Psychotherapie mehr und mehr den Psychologen überlassen wird, die eine intensivere Ausbildung auf ihrem Weg zum approbierten Psychotherapeuten erfahren. Neben allen Fortschritten der biologischen Psychiatrie sollten wir nicht vergessen, dass auch die Psychotherapie mit tiefgreifenden neurobiologischen Veränderungen einhergeht. Durch den engen Kontakt zu unseren Patienten ist sie aber häufig mit einer hohen persönlichen Befriedigung verbunden, die wir unseren jungen Ärztinnen und Ärzten nicht vorenthalten sollten. Der enge Austausch mit unseren Patienten ist vielfach ein Motiv für junge Ärzte, unsere Disziplin zu wählen.

- Ein wichtiger Punkt der Nachwuchsförderung wurde in dem Artikel von Helmut Remschmidt nicht genannt: die Frauenförderung. In der Zeit meiner Tätigkeit hat der Anteil der Frauen unter den Weiterzubildenden in der Kinder- und Jugendpsychiatrie kontinuierlich zugenommen. Er liegt heute bei ca. 80 bis $90 \%$. Trotzdem ist es mir nicht gelungen, mehr Frauen zu habilitieren, obwohl dies ein ausgemachtes Ziel zu Beginn meiner Tätigkeit war. Um zukünftig Kinder und Jugendliche mit psychischen Problemen vor allem im stationären Bereich ausreichend versorgen zu können, brauchen wir fortschrittlichere Facharztweiterbildungs- und Arbeitszeitmodelle, z.B. Schichtdienste, die größere Überlappungszeiten der klinisch tätigen Kolleginnen (und Kollegen) erlauben, 
um ggf. eine Betreuung unserer Patienten und ihrer Bezugspersonen durch zwei Therapeuten sicherzustellen. Auch die Forschungstätigkeit unserer Kolleginnen endet vielfach abrupt mit der Geburt eines Kindes. Hier kann nur durch Anerkennung von Forschungstätigkeit auf die Facharztweiterbildungszeit Abhilfe geschaffen werden. Während der letzten Jahre hat sich die Anzahl von weiblichen Lehrstuhlinhabern trotz der wesentlichen Zunahme von Assistenzärztinnen kaum erhöht. Dies sollte uns allen zu denken geben!

Ich möchte dieses Editorial nicht beenden ohne einen großen Dank für die Freundschaft und Zusammenarbeit mit meinen Mitstreitern auszudrücken; in den ersten Jahren waren dies vor allem Gerd Lehmkuhl und Andreas Warnke, mit denen ich belebende und tiefgreifende Diskussionen erinnere; in den letzten Jahren danke ich für die Toleranz und das Engagement von Martin Holtmann und Benno Schimmelmann, mit denen immer konstruktive Lösungen gefunden werden konnten. Ich wünsche ihnen und meinen Nachfolgern von Herzen ein gutes Gelingen für diese Zeitschrift, die einen wichtigen Beitrag zur „core identity“ unseres Faches liefern kann und muss.

\section{Literatur}

Bühren K., Herpertz-Dahlmann B., Dempfle A., Becker K., Egberts K. M., Ehrlich S. et al. (2017). First sociodemographic, pretreatment and clinical data from a German web-based registry for child and adolescent Anorexia Nervosa. Z Kinder Jugendpsychiatr Psychother. 45(5):393-400.

Remschmidt H. (2018). 50 Jahre Facharzt für Kinder- und Jugendpsychiatrie in Deutschland. Geschichte, Gegenwart und Zukunft Kinder Jugendpsychiatr Psychother. 46(6):466-477.

Revet A., Hebebrand J., Bhide S., Caseiro J., Conti C., Deutz M., et al. (2018). Dual training as clinician-scientist in child and adolescent psychiatry: are we there yet? Eur Child \& Adolesc Psychiat 27:263-265.

Rothenberger A. (2005). Editorial: Auf dem Weg zu einer „Europäischen Kinder- und Jugendpsychiatrie". Z. Kinder-Jugendpsychiatry. 33(4), 255-257.

Schulte-Markwort M., Lehmkuhl U. (2005). Editorial. Kinder- und Jugendpsychosomatik. Z. Kinder-Jugendpsychiatr. 33(2), 75-76.

Prof. Dr. med. Beate Herpertz-Dahlmann

Klinik für Psychiatrie, Psychosomatik

und Psychotherapie des Kindes- und Jugendalters

Neuenhofer Weg 21

52074 Aachen

Deutschland

bherpertz@ukaachen.de 